(0-16yo), place of diagnosis (local hospital), and new diagnosis.

Results 21 patients were identified within the database; four were excluded due to diagnosis in other country or lack of notes. 9 females and 8 males were included, with a mean age of 8 years and a median duration of presenting symptoms of 21 days. 82\% were referred to the paediatric emergency department by their general practitioner (GP). 82\% reported polyuria, $76 \%$ reported polydipsia, 29\% reported fatigue and weight loss, with other presenting symptoms including nocturia and high capillary blood glucose measured at home. $76 \%$ had a family history of diabetes or autoimmune disease. $82 \%$ presented with hyperglycaemia, and $18 \%$ in diabetic ketoacidosis. Mean presenting capillary blood glucose was $24 \mathrm{mmol} / \mathrm{L}$ with a mean $\mathrm{HbA} 1 \mathrm{c}$ of $106 \mathrm{mmol} / \mathrm{mol}$. Only 2 patients (12\%) had urine dipstick results documented on their hospital discharge summary. 16 subjects had a new diagnosis of type 1 diabetes and 1 had a new diagnosis of type 2 diabetes. Compared to previous data from 2013, in which 50\% of patients had a delay in diagnosis, 3 patients $(18 \%)$ had delayed diagnosis of diabetes. All of this group were seen prior to diagnosis by their GP, with a median duration of delay of 3 days.

Conclusion Delay in diagnosis of diabetes in children appears to have reduced in the past 5 years in the local area. However, practice must continue to improve to prevent unacceptable delay. This requires education to improve symptom recognition, of both local healthcare professionals, and also of adult patients with autoimmune disease, in view of the high incidence of diabetes in children with a family history.

\section{G430(P) USING THE HOSPITAL SCHOOL AS A RESOURCE TO WIDEN THE SCOPE OF CONVERSATION DURING CLINICAL DIABETES CONSULTATIONS}

${ }^{1}$ VE Dublon, ${ }^{2} S$ Green, ${ }^{1}$ R Arscott. 'Paediatric Diabetes, Royal Free Hospital NHS Trust, London, UK; ${ }^{2}$ Royal Free Hospital School, Royal Free Hospital School, London, UK

\subsection{6/archdischild-2020-rcpch.371}

Introduction Exploring the impact of diabetes on the wider life of 'school examination aged young people' is thought to be really important. We hoped that doing this 'Me First' approach in a demonstrative way would encourage greater self efficiacy in the management of both their diabetes and everyday life. At this age, preparation and completion of examinations is one of the most important elements of a young persons life, so it made sense to involve the Hospital School as part of our clinical consultations.

Method We introduced a 'meet and greet' system, which involved the young person being greeted on arrival in clinic by a hospital school teacher. They sat down immediately with the teacher, discussed school matters, also completeing an 'All About Me' form, with time to talk over their choices. The 'All About Me' form gathers personal information such as family support, likes, dislikes, goals, ambitions, social and educational needs and preferences. Young People were also given specific information regarding extra time and special consideration for exams. They then went on to see the diabetes team as usual. This method also eliminates 'the waiting aspect' to clinic.

The young people and staff were then asked to give feedback regarding the new approach to consultations.
Results

- Young people and staff reported the 'All About Me' form, gave improved depth and quality of conversation around their diabetes, putting their needs at the centre of the clinic visit from the very start.

- $100 \%$ of the diabetes team felt this led to easier flow of conversation.

- $100 \%$ of the young people were not aware that access arrangements and special consideration could be applied for on the grounds of having diabetes.

- $100 \%$ of the young people had experienced diabetes related issues around completing examinations.

- $100 \%$ of the young people requested their schools to be contacted and informed regarding access arrangements.

Conclusion Discussion around school and using the All About Me' form, clearly placed young people at the heart of the consultation, 'Me First' approach, and provided an opportuntiy for them to discuss what matters to them as well as being more able to articulate their own goals.

Young people are underinformed regarding school exam access arrangments and are eager to embrace this, consenting to their schools being informed.

\section{G431(P) GENDER DYSPHORIA - A DESCRIPTION OF THE CHANGES IN PREVALENCE, DEMOGRAPHICS AND THE CLINICAL CARE PROVIDED BY A PAEDIATRIC ENDOCRINOLOGY DEPARTMENT}

${ }^{1} \mathrm{~S}$ McCallion, ${ }^{2} \mathrm{~S}$ Smith, ${ }^{1} \mathrm{H}$ Kyle, ${ }^{1} \mathrm{~J}$ McQuarrie, ${ }^{2} \mathrm{G}$ Wilkinson, ${ }^{1} \mathrm{~A}$ Kyriakou. ${ }^{1}$ Department of Paediatric Endocrinology, Royal Hospital for Children, Glasgow, UK; ${ }^{2}$ Young Persons Gender Service, Sandyford, Glasgow, UK

\subsection{6/archdischild-2020-rcpch.372}

Aim To describe the trends in prevalence, clinical characteristics and clinic journey of young people (YP) attending the paediatric endocrine service, because of Gender Dysphoria (GD).

Methods Electronic medical records of YP attending the paediatric endocrine service, between 2011-2018, were reviewed.

Results Overall, 74 YP [49 (66\%) birth-assigned females and 25 (34\%) birth-assigned males] were assessed by paediatric endocrinology in 2011-2018. Forty-six (62\%) were referred in the 2-year period 2017-2018. Median age (range) at initial assessment was 14.6 yrs $(8.8,17.5)$ and remained unchanged during the study period $(\mathrm{p}=0.862)$. Median age (range) at initial assessment was 14.3 yrs $(8.8,15.8)$ for birth-assigned females and 15.0 yrs $(9.8,17.5)$ for birthassigned males $(p=0.096)$. There was a 4 -fold increase in the number of YP attending paediatric endocrinology from $2015(\mathrm{n}=15)$ to $2018(\mathrm{n}=60)$. Overall, 55/74 (36 birthassigned females, 19 birth-assigned males) were eligible for referral for fertility preservation, of which 18 (33\%) (12 birth-assigned females, 6 birth-assigned males, $p=1.0$ ) sought a formal referral to Fertility service. One (8\%) birthassigned female and $5(83 \%)$ birth-assigned males completed fertility preservation $(\mathrm{p}=0.004)$. Sixty-five $(88 \%)$ of those attended [44 (90\%) birth-assigned females and 21 (84\%) birth-assigned males] were commenced on gonadotrophinreleasing hormone analogue $(\mathrm{GnRHa})$ for puberty suppression. Of those, 46 (71\%) were commenced on GnRHa in 